\title{
Developing a vascular access team
}

Vicki Shawyer

When it was first created, the remit of the vascular access team at the Royal Devon and Exeter NHS Foundation Trust was to reduce the rates of intravenous (IV) access-associated complications and bacteremia by improving patient assessment and care of IV access devices.

The principle drivers of Winning Ways (Chief Medical Officer, 2003) and Saving lives (Department of Health (DH), 2005) were the need

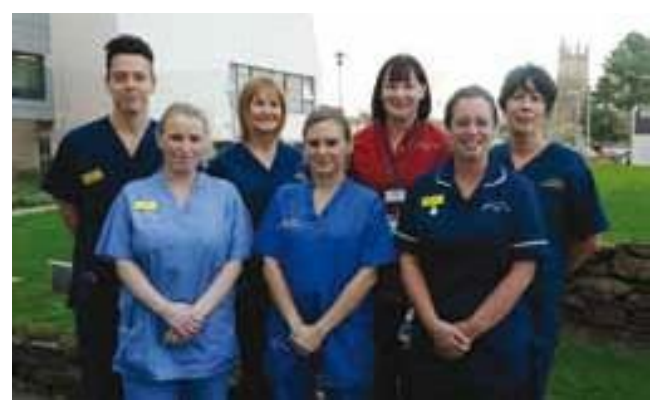
to reduce infection associated with invasive devices. With central venous catheter care one of the top five high-impact interventions, the challenge is to ensure the dissemination and application of best practice. The Health and Social Care Act 2008: Code of Practice (Department of Health, 2015) states that providers must audit compliance with key policies and procedures for infection prevention. The Care Quality Commission (CQC) assesses compliance to ensure the care people receive meets essential standards of quality and safety.

In 2008, the key challenges facing the team were: patient safety, high bed occupancy, costly antibiotics, procurement and education. Today the vascular access team comprises registered nurses and allied health professionals who have developed extensive specialist knowledge, skills and competence in effective patient assessment, bedside device insertion, maintenance and care.

The benefits of a vascular access team are well recognised both locally and nationally (Jackson, 2007; Meyer, 2012; Walker et al, 2013; Chopra et al, 2015). Such benefits can include:

- A reduction in device-related complications, including catheter-related bloodstream infections

- Increased patient satisfaction and comfort

- Timely completion of therapies

- An increased level of clinical expertise

- Use of state-of-the-art technology such as ultrasound

- A reduction in patient length of stay (e.g. internal figures show that from January to November 2015, the team saved 2859 bed days)

- Early assessment and identification of vascular access requirements.

The reason I am so proud to be part of the vascular access team can be seen in what has been achieved and the results. We now have a direct referral system making it easier for clinicians including GPs to refer patients to us. Historically, patients stayed in hospital to receive their IV antibiotic treatment, now they are discharged to home or attend their local community hospital as outpatients to receive IV antibiotics. For example, our cardiology patients with endocarditis previously required a 6-week inpatient stay to receive IV antibiotics. These patients can now be discharged home with a peripherally inserted central catheter (PICC) to be looked after by the community IV team. Similarly, patients with respiratory infections who previously needed to spend 2 weeks as inpatients now see us to have a midline inserted and receive IV antibiotics at home, while patients with infected joints also benefit from prompt access and reduced length of stay. For our haematology and oncology patients we now carry out line insertion, and chemotherapy is started on the same day to save time for the patient. There is also a dedicated list using ultrasound to take blood samples from patients with difficult venous access. This includes IV drug users, who previously could not access treatment for hepatitis, which requires blood monitoring, as staff were unable to obtain samples (owing to the poor condition of their veins). 
In 2009, the rate of bacteraemias started increasing - this was associated with administration of parenteral nutrition (in 2008, the rate was 12.25 per 1000 days). The vascular access team worked in collaboration with the learning and development service, nutrition support team, and infection control team to implement changes, and the rate has dropped to 0.63 per 1000 days. This was accomplished by, for example:

- Same-day referral for line insertion to prevent insertion of short-term central venous catheters and associated risks

- Parenteral competencies and care plans developed with training

- Only staff trained and assessed as competent are allowed to set up and disconnect parenteral nutrition, with an yearly refresher.

The increased use of PICCs following the appointment of the vascular access team in 2008 led to an increase in PICC-associated bacteremia. This was found to be the result not of poor insertion technique, but of problems with ongoing care of the lines. A robust programme of training for ward staff was implemented in 2009 and continues for any staff accessing any CVC. This was associated with a significant reduction in the bacteremia rate from $2.6 \%$ to $0.08 \%$.

To describe what we do in one day is impossible. Our days are varied by the different people, teams and areas that we interact and engage with daily. What stays the same is our focus on providing a first class service for our patients and staff.

Chopra V, Flanders SA, Saint S et al (2015) The Michigan Appropriateness Guide for Intravenous Catheters (MAGIC): Results From a Multispecialty Panel Using the RAND/UCLA Appropriateness Method. Ann Intern Med 163(6 Suppl): S1-40

Chief Medical Officer (2003) Winning Ways. Working Together to Reduce Healthcare Associated Infection in England. http://tinyurl.com/na4q8bs (accessed 13 January 2016)

Department of Health (2005) Saving Lives: delivery programme to reduce healthcare-associated infection (HCAl) including MRSA. Department of Health, London

Department of Health (2010) The Health and Social Care Act 2008: Code of practice for health and adult social care on the prevention and control of infections and related guidance. http://tinyurl.com/p3dr4hg (accessed 19 January 2016)

Walker G,Todd A (2013) Nurse-led PICC insertion: is it cost effective? Br J Nurs 22(19Suppl): S9-15

Jackson A (2007) Development of a trustwide vascular access team. Nurs Times 103: 44 28-9

Meyer BM (2012) Developing an alternative workflow model for peripherally inserted central catheter placement. J Infus Nurs 35(1): 34-42 